\title{
Instabilities and quasi-localized states in nonlinear Fano-like systems
}

\author{
Andrey E. Miroshnichenko \\ Nonlinear Physics Centre and Centre for Ultra-high bandwidth Devices for Optical Systems (CUDOS), Australian National University, Canberra ACT 0200, Australia
}

\section{A R T I C L E I N F O}

\section{Article history:}

Received 28 December 2008

Received in revised form 4 February 2009

Accepted 5 February 2009

Available online 3 August 2009

Communicated by V.M. Agranovich

\section{Keywords:}

Fano resonance

Modulational instability

Quasi-localized states

\begin{abstract}
A B S T R A C T
We study the dynamical scattering in one-dimensional systems with a nonlinear side-coupled defect. Such structures exhibit the nonlinear Fano resonances, where nothing can propagate through. We developed a numerical model to study dynamical scattering. According to our analysis the scattering waves become dynamically unstable in the vicinity of the nonlinear Fano resonances, due to modulational instability caused by the presence of nonlinearity. It results in a time-growing amplitude of the nonlinear defect. We also demonstrate the existence of the nonlinear quasi-localized state, supported by such structures.
\end{abstract}

(c) 2009 Elsevier B.V. All rights reserved.

\section{Introduction}

It is well known that presence of defects in physical systems may lead to appearance of resonances in the transmission. Usually, they are characterized by an enhancement of the transmission. But, there are such structures where defects may lead to the resonant suppression of the transmission (or resonant reflection). Such kind of phenomena are widely known across various branches of physics as the Fano resonances, first explained by Ugo Fano in application to the atom ionization problem [1]. The underlying physics of the phenomenon is the quantum interference of several processes, which can be mapped to wave interference in the scattering problem, where the co-existence of at least two propagating paths for scattering waves leads to constructive and destructive interferences. Such a simple picture can be easily extended to many other systems, which makes the Fano resonance a quite universal phenomenon. Fano has derived a very simple formula to describe an asymmetric profile, associated with Fano resonances. Fano resonances were observed in various systems such as, for example, propagation of electrons in systems with quantum dots [2,3], and their application to spin filters [4], light propagation in photonic crystal circuits [5-14], scattering of phonons by discrete breathers (or time-periodic scattering potentials) [15-17], scattering of atoms in Bose-Einstein condensate in an optical lattice [18-20], Mie resonances and light scattering by nanoparticles [21] and etc.

Recently, we have studied $[22,23]$ nonlinear Fano resonances in one-dimensional linear systems with a nonlinear side-coupled defect. It was demonstrated, that it is possible to completely sup-

E-mail address: aem124@rsphysse.anu.edu.au. press the transmission for certain values of intensity of the incoming wave. It provides with tunability of the Fano resonances. Together with the bistable behavior is was successfully applied in photonic crystal circuits to achieve all-optical switching operation $[7,8,10-13]$. The advantage of the nonlinear Fano resonance is the possibility of the total $100 \%$ switching, which was recently demonstrated in Ref. [14].

Up to now, the nonlinear Fano resonance was analyzed in the steady-state regime only, where the single-frequency scattered waves propagating in a system were assumed. Usually, this assumption is enough to describe scattering properties of physical systems. But, as it was shown by Malomed and Azbel [24], in general, nonlinear defects may lead to generation of additional frequencies, due to nonlinear mechanism, resulting in modulational instability. In such a case there will co-exist scattered waves with incommensurate frequencies, who's superposition will lead to time dependent transmission and reflection coefficients. Naturally, the question arises about applicability of results of steady-state analysis in nonlinear systems.

The aim of this Letter is to study the full dynamical scattering process by a nonlinear Fano defect without any assumptions. For that purpose we've developed the special numerical method, allowing us to study the problem with a great precision. In particular, we demonstrate that in the vicinity of the nonlinear Fano resonances scattered waves become dynamically unstable with time-growing amplitudes at the nonlinear side-coupled defect. The spectral analysis reveals the presence of several frequencies in the dynamics. Surprisingly, both stable and unstable regimes contain the exactly same frequency in addition to the pumping one. To address the nature of this spurious frequency, we have performed an additional analysis, and discovered the existence of quasi-localized state, supported by the nonlinear Fano defect. This state persists 


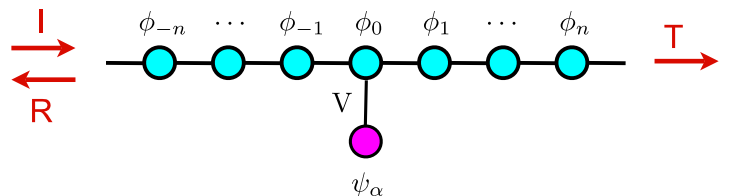

Fig. 1. (Color online.) Schematic view of a one-dimensional system with a nonlinear side-coupled Fano defect (1).

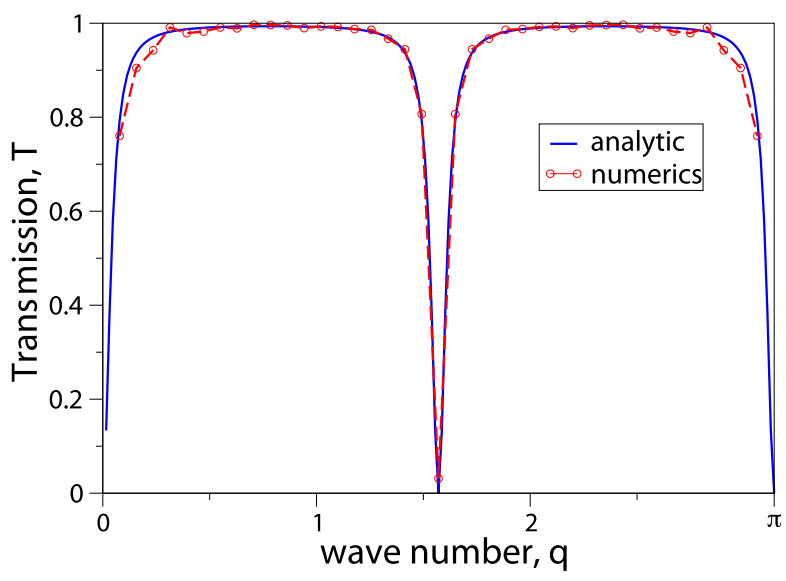

Fig. 2. (Color online.) Comparison of the linear transmission coefficient obtained analytically [22] and numerically from the system (14). The parameters are $V=0.8$, $E_{F}=0$, and $\lambda=0$.

for very long time without any change of its profile, which allows us to conclude that it is one of the solutions of the system.

The Letter is organizes as follows. In Section 2 the dynamical model is introduced. Section 3 is devoted to the stability analysis. In Section 4 the developed numerical method is described in details. All results are presented and discussed in Section 5. Finally, Section 6 concludes the Letter.

\section{Model}

We investigate the dynamical scattering problem of continuous waves (CWs) in one-dimensional linear system with a single sidecoupled nonlinear defect (see Fig. 1)

$i \dot{\phi}_{n}=\left(\phi_{n-1}+\phi_{n+1}\right)+\psi_{\alpha} V \delta_{n 0}$,

$i \dot{\psi}_{\alpha}=\lambda\left|\psi_{\alpha}\right|^{2} \psi_{\alpha}+V \phi_{0}$,

where we consider a Kerr type of nonlinearity with coefficient $\lambda$, together with the following boundary conditions

$\phi_{n}=\mathrm{e}^{-\mathrm{i} \omega_{q} t} \begin{cases}I \mathrm{e}^{\mathrm{i} q n}+r \mathrm{e}^{-\mathrm{i} q n}, & n<0, \\ t \mathrm{e}^{\mathrm{i} q n}, & n>0\end{cases}$

with the dispersion $\omega_{q}=2 \cos q$.

Usually, to solve the scattering problem (1) the steady-state solution is considered, by eliminating time-dependency using the substitution

$\phi_{n}(t)=A_{n} \mathrm{e}^{-i \omega_{q} t}$,

$\psi_{\alpha}(t)=B \mathrm{e}^{-i \omega_{q} t}$.

The complete analytical solution of the system (1) with the static ansatz (3) can be found in Ref. [22]. It was shown that the system (1) may exhibit so-called nonlinear Fano resonances, where the transmission coefficient $T=|t / I|^{2}$ completely vanishes $T=0$ for certain relations of the wavenumber $q$ and power $P=|I|^{2}$. The typical Fano profile in the linear regime $\lambda=0$ is shown in Fig. 2.
As it was shown by Malomed and Azbel [24] nonlinear resonances, in general, may exhibit dynamical (or modulational) instabilities, where additional frequencies can be generated via nonlinear interactions. As a result, the general solution of the system (1) might not be described by the steady-state ansatz (3).

In this Letter we study the full dynamical scattering problem (1), (2) without any assumptions by using numerical method, described below.

\section{Stability analysis}

As the first step of our study, we perform stability analysis of steady-state solutions, which can be found in Ref. [22]. We linearize the system (1) around the given static solution $\left\{\phi_{n}, \psi_{\alpha}\right\}$ by adding a small perturbation $\left\{\varepsilon_{n}, \kappa\right\}$

$\mathrm{i} \dot{\varepsilon}_{n}=C\left(\varepsilon_{n-1}+\varepsilon_{n+1}\right) V \kappa \delta_{n 0}$,

$\mathrm{i} \dot{\kappa}=2 \lambda\left|\psi_{\alpha}\right|^{2} \kappa+\lambda \psi_{\alpha}^{2} \kappa^{*}+V \varepsilon_{0}$,

where ${ }^{*}$ denotes complexion conjugation. Note here, that the linearized system (4) has zero boundary conditions.

By using the fact that steady-state solutions has the form (3), we can eliminate time in the linearized system (4) by the following substitutions

$\varepsilon_{n}(t)=a_{n} \mathrm{e}^{-i \omega t}+b_{n}^{*} \mathrm{e}^{i\left(\omega^{*}-2 \omega_{q}\right) t}$,
$\kappa(t)=c \mathrm{e}^{-i \omega t}+d^{*} \mathrm{e}^{i\left(\omega^{*}-2 \omega_{q}\right) t}$,

where $\omega$ is a frequency of small amplitude fluctuations and $\omega_{q}$ is the frequency of the incoming wave. It provides us with the correctly defined eigenvalue problem

$\omega a_{n}=a_{n-1}+a_{n+1}+V c \delta_{n 0}$,

$-\omega b_{n}=b_{n-1}+b_{n+1}-2 \omega_{q} b+V d \delta_{n 0}$,

$\omega c=2 \lambda|B|^{2} c+\lambda B^{2} d+V a_{0}$,

$-\omega d=2 \lambda|B|^{2} d-2 \omega_{q} d+\lambda_{d} B^{2} c+V b_{0}$.

According to our ansatz (5), the initial nonlinear state $\left\{\phi_{n}, \phi_{\alpha}\right\}$ is dynamically unstable, when there is at list one eigenvalue $\omega$ such that $\operatorname{Im}(\omega)>0$, resulting in the solution whose amplitude is growing in time.

Fig. 3 shows typical nonlinear Fano resonance with corresponding results of the stability analysis, indicating that, in the vicinity of the nonlinear resonance (suppression of the transmission) the steady-state solution becomes dynamically unstable.

\section{Numerical approach}

Since it is impossible to get analytical solution of the system (1), which will describe the complete dynamics, we need to solve it numerically. For that purpose, we developed a special numerical method.

We may consider the incoming CW wave as an external force, due to its known analytical representation, and write the boundary conditions in the following general form

$\phi_{n}(t)= \begin{cases}I \mathrm{e}^{\mathrm{i} q n-\mathrm{i} \omega_{q} t}+\phi_{n}^{R}(t), & n<0, \\ \phi_{n}^{T}(t), & n>0,\end{cases}$

where $\phi_{n}^{R}(t)$ and $\phi_{n}^{T}(t)$ correspond to the reflected and transmitted waves.

By using the fact that far away from the nonlinear Fano defect $|n| \gg 0$ the system is linear and free of the defect, we get the following set of equations for 


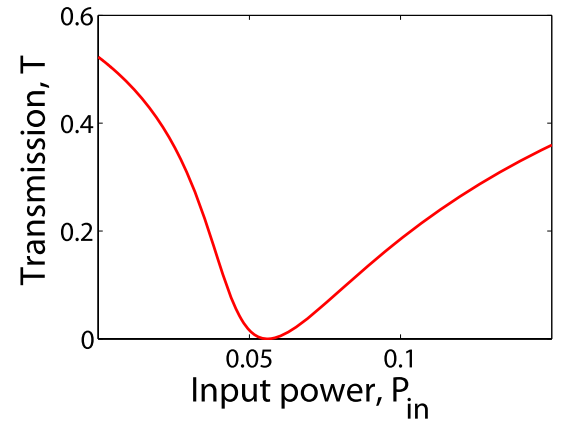

(a)

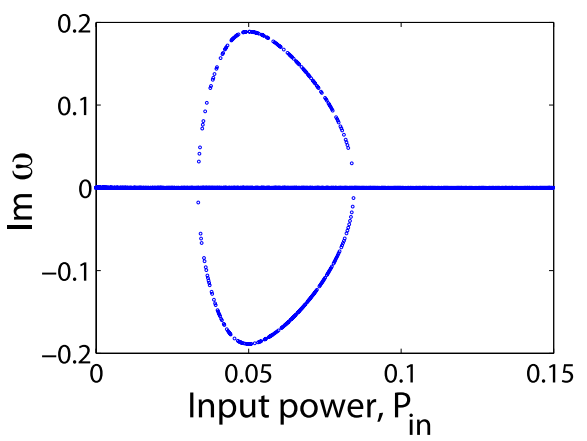

(b)

Fig. 3. (Color online.) (a) Nonlinear transmission coefficient, and (b) corresponding imaginary parts of the eigenvalues of the system (6) vs input power. The parameters are $V=0.8, \lambda=1$, and $q=1.4$ (see Fig. 2).

(a) transmitted, $n>0$

$$
i \dot{\phi}_{n}^{T}=\phi_{n-1}^{T}+\phi_{n+1}^{T},
$$

(b) and reflected waves only, $n<-1$

$$
\begin{aligned}
& \omega I \mathrm{e}^{\mathrm{i} q n-\mathrm{i} \omega t}+i \dot{\phi}_{n}^{R} \\
& \quad=\left(\mathrm{e}^{\mathrm{i} q}+\mathrm{e}^{-\mathrm{i} q}\right) I \mathrm{e}^{\mathrm{i} q n-\mathrm{i} \omega t}+\phi_{n-1}^{R}+\phi_{n+1}^{R} .
\end{aligned}
$$

After substitution $\omega=2 \cos q=\mathrm{e}^{\mathrm{i} q}+\mathrm{e}^{-\mathrm{i} q}$ into (9), the external force I vanishes completely, and the equation for the reflection wave, $n<-1$, takes the same form, as for transmitted one (8)

$i \dot{\phi}_{n}^{R}=\phi_{n-1}^{R}+\phi_{n+1}^{R}$.

As a result the external force $I$ will be explicitly included only at two sites $n=-1$ and $n=0$ :

(c) for $n=-1$

$$
\omega I \mathrm{e}^{-\mathrm{i} q-\mathrm{i} \omega t}+i \dot{\phi}_{-1}^{R}=\phi_{-2}^{R}+\phi_{0}^{T}+I \mathrm{e}^{-2 \mathrm{i} q-\mathrm{i} \omega t},
$$

which can be simplified to

$$
i \dot{\phi}_{-1}^{R}=\phi_{-2}^{R}+\phi_{0}^{T}-I \mathrm{e}^{-\mathrm{i} \omega t},
$$

(d) and the final equation for $n=0$

$$
i \dot{\phi}_{0}^{T}=\phi_{-1}^{R}+\phi_{1}^{T}+I \mathrm{e}^{-\mathrm{i} q-\mathrm{i} \omega t}+V \psi_{\alpha},
$$

where the continuity condition for site $n=0$ was used $\phi_{0} \equiv$ $\phi_{0}^{T}=I \mathrm{e}^{-\mathrm{i} \omega t}+\phi_{0}^{R}(7)$.

Now we can omit all superscripts and write down the new set of equations for dynamical scattering problem

$$
\begin{aligned}
& i \dot{\phi}_{n}=\phi_{n-1}+\phi_{n+1}, \quad n<-1, n>0, \\
& i \dot{\phi}_{-1}=\phi_{-2}+\phi_{0}-I \mathrm{e}^{-\mathrm{i} \omega t}, \\
& i \dot{\phi}_{0}=\phi_{-1}+\phi_{1}+I \mathrm{e}^{-\mathrm{i} q-\mathrm{i} \omega t}+V \psi_{\alpha}, \\
& i \dot{\psi}_{\alpha}=\lambda\left|\psi_{\alpha}\right|^{2} \psi_{\alpha}+V \phi_{0},
\end{aligned}
$$

where $\phi_{n}$ describes the reflected waves for $n<0$ and transmitted waves for $n \geqslant 0$.

As was mentioned above, due to modulational instability in the vicinity of the nonlinear Fano resonance, additional frequencies may be generated in the full dynamical problem (14). It will require special type of boundary conditions, which will absorb any radiation, and reflect none. We found that discrete transparent boundary conditions method [25] is very suitable for our problem. The comparison of analytical and numerical results in the linear regime is shown in Fig. 2, demonstrating the perfect agreement. This method allows us to take only few number of sites, and, thus, drastically optimize the computation time.

\section{Results and discussions}

In this section we present our numerical results on dynamical scattering of CWs by the nonlinear side-coupled defect. In addition to that we demonstrate the existence of quasi-localized state in such a system.

\subsection{Modulational instability}

According to stability analysis in Section 3 it turned out that CWs excitation become dynamically unstable in the vicinity of the nonlinear Fano resonances [see Fig. 3]. As a result, we may expect that the system will diverge in time. By using our numerical method, developed in Section 4, we are going to verify this result numerically.

Fig. 4 shows results for two values of incoming power: at resonance $P_{\text {in }}=0.056$ [see Fig. $4(\mathrm{a}, \mathrm{b})$ ] and out of resonance $P_{\text {in }}=$ $2.5 \times 10^{-3}$ [see Fig. 4(c, d)]. One can clearly see, that the amplitude of the nonlinear side-coupled defect is diverging in time (see Fig. 4(a)) at the resonance, while out of resonance that system is reaching the steady-state regime [see Fig. 4(c)]. Despite the fact that there is a modulational instability in the system, the transmission coefficient is still very well defined [see Fig. 4(b, d)], and corresponds to its steady-state value [see Fig. 3(a)].

One may notice, that even out of resonance there are some beatings in the dynamics of the transmission coefficient [see Fig. 4(d)], indicating that even in this case several frequencies are present in the dynamics. To clarify this issue, we analyze a Fourier spectrum of the dynamics of the nonlinear side-coupled defect [see Fig. 5]. Indeed, in both cases there are two main frequencies: the pumping one $\omega_{q}$, and an additional one $\Omega_{0}=0.2$. The only difference between two spectra is the presence of two small satellites $\Omega_{1}$ and $\Omega_{-1}$ near $\Omega_{0}$ frequency, which correspond to frequencies of the unstable modes, and are responsible for modulational instability. It indicates that the frequency $\Omega_{0}$ plays an important role in the dynamics, and its nature will be investigated in the next Section.

\subsection{Quasi-localized mode}

The appearance of the same additional frequency $\Omega_{0}$ in dynamics of both regimes indicates that is somehow related to the internal dynamics of the nonlinear defect. To realize what kind of dynamical states are possible to excite in our system, we perform the calculation of the spatial Gaussian pulse propagation of the following form

$\phi_{n}(0)=I_{0} \exp \left(\mathrm{i} q n-\left(n-n_{0}\right)^{2} / \sigma^{2}\right)$.

If the maximum power of the spatial Gaussian $P=\left|I_{0}\right|^{2}$ is larger than a certain threshold, nonlinear excitations can be generated. Indeed, our results demonstrate that some nonlinear excitation persists for very long time without changing its profile [see 


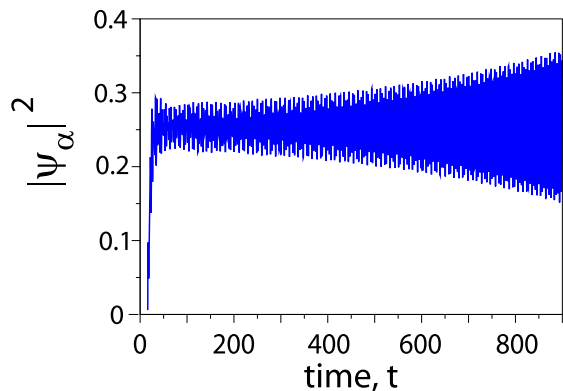

(a)

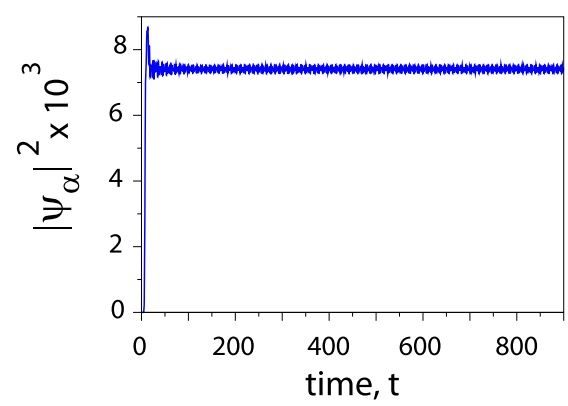

(c)

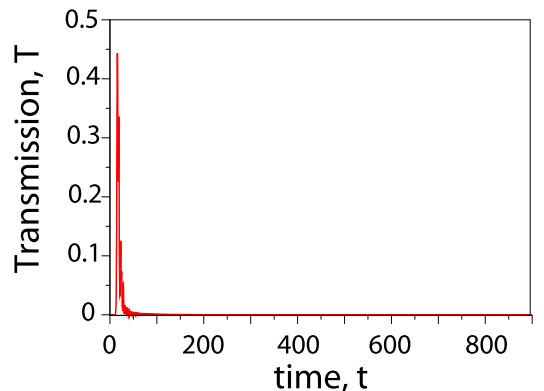

(b)

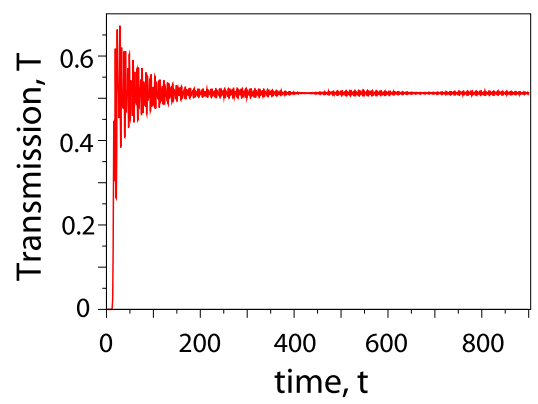

(d)

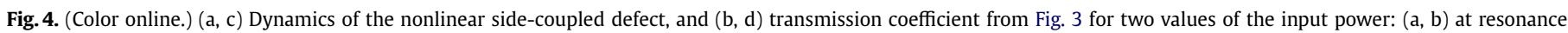
$P_{\text {in }}=0.056$, and $(\mathrm{c}, \mathrm{d})$ out of resonance $P_{\text {in }}=2.5 \times 10^{-3}$.

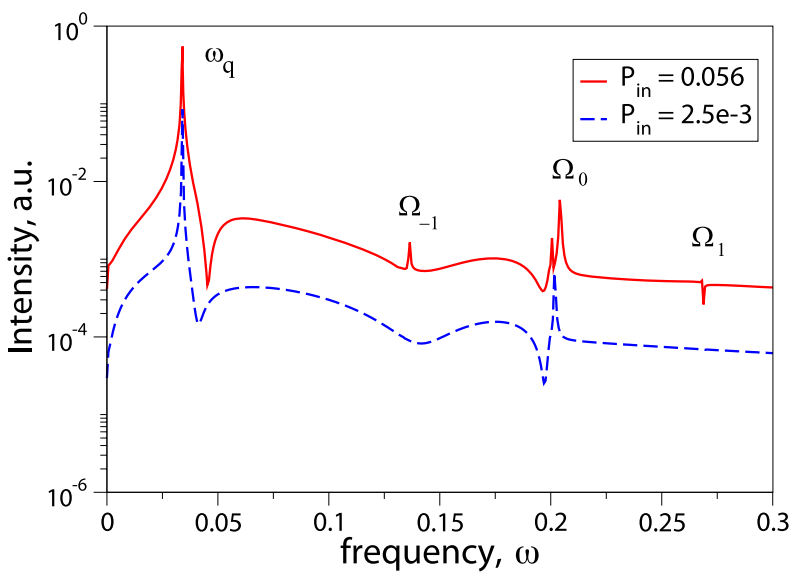

Fig. 5. (Color online.) Fourier spectrum corresponding to the dynamics of the nonlinear side-coupled defect shown in Fig. 4(a) (solid, red), and Fig. 4(c) (dashed, blue).

Fig. 6(a)]. But, this is not a nonlinear localized mode of the sidecoupled defect, because it spatial profile is not exponentially localized [see Fig. 6(b)]. Moreover, the Fourier spectrum of it's dynamics (see Fig. 7) reveals that there is one well pronounced frequency $\Omega=0.4$ (which lies inside the propagation spectrum of the system $\Omega<2$ ) together with some other less pronounced peaks. It allows us to call this self-sustained mode as a quasi-localized mode with quasi-periodic dynamics. Thus, such type of quasi-localized mode is one the solutions of the system (1).

One might note, that exactly one of its harmonics $\Omega_{0}=\Omega / 2$ exists in the dynamics of CWs scattering (see Fig. 5). All of these allows us to suggest that this unusual quasi-localized state is always excited in dynamical scattering process. Moreover, it is responsible for modulational instability at the resonances.

\section{Conclusions}

We have studied the wave scattering by the nonlinear sidecoupled Fano defect in one-dimensional systems. The system ex-

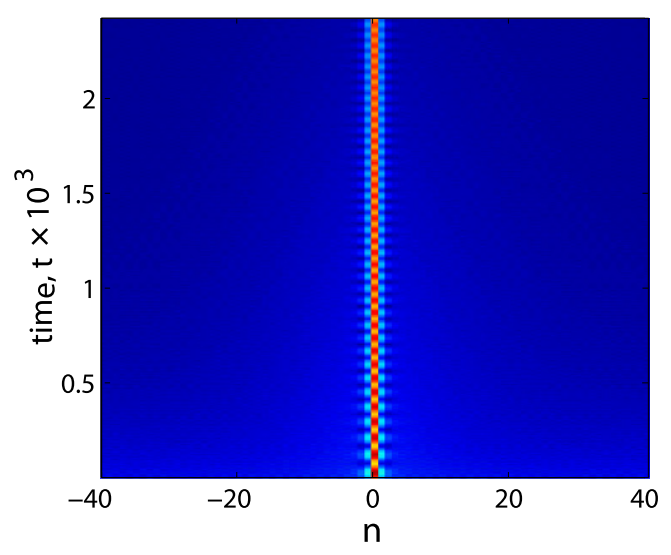

(a)

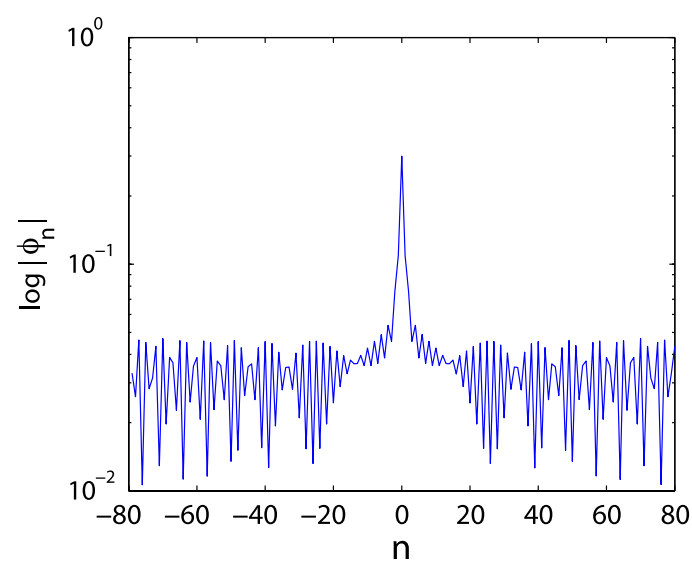

(b)

Fig. 6. (Color online.) (a) Spatio-temporal plot of the system dynamics after propagation of the input Gaussian pulse (15). (b) Spatial profile at time $t=150$. The parameters of the Gaussian pulse are $I_{0}=10$, and $\sigma=14.14$. 


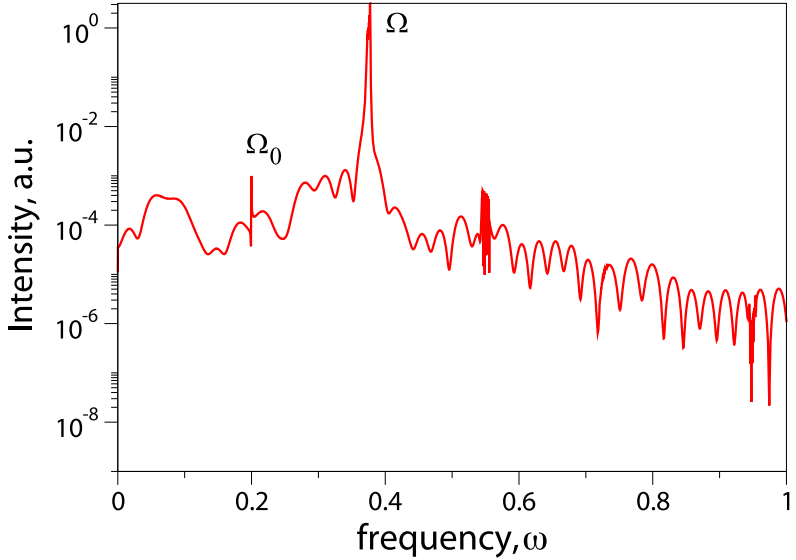

Fig. 7. (Color online.) Fourier spectrum of the quasi-localized mode shown in Fig. 6.

hibits total suppression of the transmission in steady-state regime, which can be associated with the nonlinear Fano resonance. By performing the stability we discovered that scattered waves may become dynamically unstable in the vicinity of the nonlinear Fano resonance, due to modulational instability, results in the time growing amplitude of the nonlinear defect. This result was confirmed numerically by using specially developed numerical method with transparent boundary conditions. Indeed, in the vicinity of the nonlinear Fano resonance, where the transmission goes to zero, the intensity of the side-coupled defect diverges with time. Moreover, we've observed dynamical spectra in stable and unstable regimes contain a spurious frequency in addition to the pumping one. Our further analysis suggests that this frequency originates from the excitation of a quasi-localized defect mode, supported by the nonlinear Fano defect. This new quasi-localized state exists for a very long time, according to our simulation results. This is quite unexpected phenomenon for such systems, where only extended or localized state may live indefinitely long, and deserves a proper and more elaborated analysis, which will be done in our future publications.

\section{Acknowledgements}

The author thanks Yuri Kivshar for useful discussions. This work was supported by Australian Research Council.

\section{References}

[1] U. Fano, Phys. Rev. 124 (1961) 1866.

[2] J. Göres, D. Goldhaber-Gordon, S. Heemeyer, M.A. Kastner, H. Shtrikman, D. Mahalu, U. Meirav, Phys. Rev. B 62 (2000) 2188.

[3] K. Kobayashi, H. Aikawa, S. Katsumoto, Y. Iyte, Phys. Rev. Lett. 88 (2002) 256806.

[4] M.E. Torio, K. Hallberg, S. Flach, A.E. Miroshnichenko, M. Titov, Eur. Phys. J. B 37 (2004) 399;

A.A. Aligia, L.A. Salguero, Phys. Rev. B 70 (2004) 075307.

[5] S.H. Fan, Appl. Phys. Lett. 80 (2002) 908.

[6] S.H. Fan, J.D. Joannopoulos, Phys. Rev. B 65 (2002) 235112.

[7] M.F. Yanik, S.H. Fan, M. Soljačić, Appl. Phys. Lett. 83 (2003) 2739.

[8] A.R. Cowan, J.F. Young, Phys. Rev. E 68 (2003) 046606.

[9] S.H. Fan, W. Suh, J.D. Joannopoulos, J. Opt. Soc. Am. A 20 (2003) 569.

[10] V. Lousse, J.P. Vigneron, Phys. Rev. B 69 (2004) 153107.

[11] A.E. Miroshnichenko, Yu.S. Kivshar, Opt. Express 13 (2005) 3969.

[12] S.F. Mingaleev, A.E. Miroshnichenko, Yu.S. Kivshar, K. Busch, Phys. Rev. E 74 (2006) 046603.

[13] S.F. Mingaleev, A.E. Miroshnichenko, Yu.S. Kivshar, Opt. Express 15 (2007) 12380.

[14] S.F. Mingaleev, A.E. Miroshnichenko, Yu.S. Kivshar, Opt. Express 16 (2008) 11647.

[15] E. Tekman, P.F. Bagwell, Phys. Rev. B 48 (1993) 2553.

[16] S.W. Kim, S. Kim, Phys. Rev. B 63 (2001) 212301.

[17] S. Flach, A.E. Miroshnichenko, V. Fleurov, M.V. Fistul, Phys. Rev. Lett. 90 (2003) 084101.

[18] R.A. Vicencio, J. Brand, S. Flach, Phys. Rev. Lett. 98 (2007) 184102.

[19] A. Micheli, A.J. Daley, D. Jakschm, P. Zoller, Phys. Rev. Lett. 93 (2004) 140408.

[20] A. Micheli, P. Zoller, Phys. Rev. A 73 (2006) 043613.

[21] M.I. Tribelsky, S. Flach, A.E. Miroshnichenko, A.V. Gorbach, Yu.S. Kivshar, Phys. Rev. Lett. 100 (2008) 043903.

[22] A.E. Miroshnichenko, S.F. Mingaleev, S. Flach, Yu.S. Kivshar, Phys. Rev. E 71 (2005) 036626.

[23] A.E. Miroshnichenko, Yu.S. Kivshar, Phys. Rev. E 72 (2005) 056611.

[24] B.A. Malomed, M.Ya. Azbel, Phys. Rev. B 47 (1993) 10402.

[25] A. Arnold, M. Ehrhardt, I. Sofronov, Commun. Math. Sci. 1 (2003) 501. 